\title{
Individualized Prediction Of Metastatic
}

\section{Involvement Of Lymph Nodes Posterior To The Right Recurrent Laryngeal Nerve In Papillary Thyroid Carcinoma}

This article was published in the following Dove Press journal: OncoTargets and Therapy

\author{
Jiang Zhu' \\ Rui Huang ${ }^{2}$ \\ DaiXing $\mathrm{Hu}^{\prime}$ \\ Yi Dou' \\ HaoYu Ren' \\ ZhiXin Yang' \\ Chang Deng' \\ Wei Xiong' \\ Denghui Wang' \\ Yu Mao' \\ Xuesong $\mathrm{Li}^{1}$ \\ XinLiang Su (DD \\ 'Department of Endocrine and Breast \\ Surgery, The First Affiliated Hospital of \\ Chongqing Medical University, Chongqing \\ 4000 16, People's Republic of China; \\ ${ }^{2}$ Department of Anesthesiology, The \\ First Affiliated Hospital of Chongqing \\ Medical University, Chongqing 400016, \\ People's Republic of China
}

\begin{abstract}
Purpose: We aimed to establish a prediction model based on preoperative clinicopathologic features and intraoperative frozen section examination for precise prediction of metastatic involvement of lymph nodes posterior to the right recurrent laryngeal nerve (LN-prRLN) in patients with papillary thyroid carcinoma (PTC).

Methods: Clinicopathologic data pertaining to patients with PTC who underwent initial thyroid surgery between July 2015 and December 2017 were collected from electronic medical records. Multivariate logistic regression analysis was performed to identify independent predictors of LN-prRLN metastasis for incorporation in the nomogram. The performance of the model was assessed using discriminative ability, calibration, and clinical application.
\end{abstract}

Results: A total of 592 patients were enrolled in this study. The LN-prRLN metastatic positivity was $19 \%$ (95\% confidence interval [CI], 15.61-21.89\%). On multivariate analysis, ultrasonography-reported LN status, extrathyroid extension, Delphian lymph node metastasis, and number of metastatic pretracheal and paratracheal lymph nodes were independent predictors of LN-prRLN metastasis. The nomogram showed good discriminative ability (C-index: 0.87; [95\% CI, 0.84-0.91]; bias-corrected C-index: 0.86 [through bootstrapping validation]) and was well calibrated. The decision curve analysis indicated potential clinical usefulness of the nomogram.

Conclusion: This study demonstrates that the risk of LN-prRLN metastasis in individual patients can be robustly predicted by a nomogram that integrates readily available preoperative clinicopathologic features and intraoperative frozen section examination. The nomogram may facilitate intraoperative decision-making for patients with PTC.

Keywords: papillary thyroid carcinoma, lymph node metastasis, lymph nodes posterior to the right recurrent laryngeal nerve, predict model, nomogram

\section{Introduction}

Globally, an estimated 567,000 incident cases of thyroid cancer were reported in 2018 . The number of new cases of thyroid cancer increased by $50 \%$ in the period 2006-2016. ${ }^{1,2}$ Papillary thyroid cancer (PTC) accounts for approximately 95\% of all thyroid cancers. PTC is characterized by a propensity for dissemination to cervical lymph nodes at an early stage; the reported rates of cervical lymph node metastasis at the time of diagnosis range from $30 \%$ to $90 \%$, even in patients with small tumors. ${ }^{3}$
Department of Endocrine and Breast Surgery, The First Affiliated Hospital of Chongqing Medical University, I Youyi

Road, Chongqing 4000I6, People's

Republic of China

Tel +86 I350830 916I

Fax +862389011463

Email suxinliang@2Icn.com 
Lymph node metastasis is associated with locoregional recurrence and distant metastasis. ${ }^{4-6}$ Despite its excellent prognosis because of good surgical outcomes, disease recurrence rates may approach $30 \%$, typically in the central compartment, and this is largely attributed to incomplete initial surgical resection. ${ }^{7}$ It is worth mentioning that the left recurrent laryngeal nerve courses along the esophagus and trachea while the right recurrent laryngeal nerve ascends through the right central compartment due to anatomical differences. Therefore, the paratracheal lymph nodes in the right central compartment are divided into two parts: lymph nodes located posterior to the right recurrent laryngeal nerve (LN-prRLN) and lymph nodes located anterior to the right recurrent laryngeal nerve. ${ }^{8}$ The LN-prRLN are distributed deep in a long, narrow, confined space; this special and complex anatomical position contains important nerves, vessels, and other important structures, which makes it challenging to perform surgical exposure and dissection. Therefore, dissection of LN-prRLN remains contentious. The arguments against LN-prRLN dissection cite this as the most difficult and risky procedure with an increased risk of devascularization of parathyroid gland and recurrent laryngeal nerve injury than other subgroups; these adverse events have a longterm effect on the quality of life of patients. ${ }^{9}$ The proponents argue that the LN-prRLN dissection during the initial operation would eliminate the cancer. Failure to remove the involved lymph nodes will result in recurrent disease. Furthermore, reoperation in this region is a surgical challenge and may cause much more adverse effects than ever before. ${ }^{10}$

Therefore, precise assessment of LN-prRLN metastasis is crucial for personalized treatment to optimally balance the dissection-related benefits and risks in patients with PTC. Accuracy of preoperative ultrasound in the central neck compartment is unsatisfactory. ${ }^{11}$ Several studies have preliminarily explored the risk factors for LN-prRLN metastasis. ${ }^{12-15}$ Thus, it is important to develop an evaluation tool adapted to the profile of an individual patient in order to accurately estimate the risk of LN-prRLN metastasis. This study aimed to develop an intraoperative decision-making predictive model based on preoperative clinicopathologic features and intraoperative frozen section examination to predict the individual risk of LN-prRLN metastasis and to improve treatment decision-making.

\section{Methods}

Data pertaining to 1258 consecutive patients with PTC who underwent thyroid surgery between July 2015 and December 2017 at the First Affiliated Hospital, Chongqing Medical
University, were retrieved from the electronic medical records. The inclusion criteria were: intraoperative frozen biopsy and postoperatively proven PTC; and patients with right-sided or bilateral lesions. The exclusion criteria were: patients who underwent reoperation; solitary lesions located in the isthmus; vocal cord paralysis examined by preoperative laryngoscopy; distant metastasis; and incomplete clinical information.

Data pertaining to the following clinicopathologic variables were collected: sex, age, tumor size, tumor location, extrathyroidal extension, multifocality, Hashimoto thyroiditis, and lymph node metastasis. Tumor location, size, and presence of lymph node metastasis were described in the preoperative ultrasonography report. Presence of extrathyroidal extension was recorded in preoperative ultrasonography report or intraoperative findings.

\section{Surgical Procedures}

At our center, for patients with right PTC with tumor size $<4 \mathrm{~cm}$, we routinely perform at least unilateral lobectomy and isthmus resection with ipsilateral central compartment neck dissection; total thyroidectomy is performed if intraoperative frozen section examination reveals metastatic involvement of lymph nodes. For patients with bilateral PTC, we perform total thyroidectomy with bilateral central compartment neck dissection. In addition, lateral lymph node dissection is considered in patients with suspected lateral lymph node metastasis based on preoperative ultrasonography or intraoperative exploration.

The boundaries of right central neck compartment were defined by the hyoid bone superiorly, the innominate artery inferiorly, the trachea medially, and the right carotid arteries laterally. In the right paratracheal basins, the superficial nodes anterior to the right recurrent laryngeal nerve were labeled as paratracheal lymph nodes in order to distinguish these from LN-prRLN ${ }^{12}$ The right central lymph nodes (rCLN) excluding the LN-prRLN refer to the group of Delphian, pretracheal, and paratracheal lymph nodes collectively. The surgical procedure was described in our previous study. ${ }^{16}$ After thyroid lobectomy, we subdivided the right central compartment into Delphian, pretracheal, paratracheal, and LN-prRLN groups depending on the anatomical position ${ }^{12}$ Four subgroups were removed and labeled sequentially, and then immediately sent for intraoperative frozen section examination. Postoperative histopathologic examination of all resected specimens was conducted independently by three pathologists. Pathologic features including tumor size, lymph 
node metastasis, and chronic lymphatic thyroiditis were recorded in the pathology report.

\section{Statistical Analysis}

The independent-samples $t$-test or Mann-Whitney $U$-test was used to compare continuous variables. Categorical variables were compared using the Chi-squared test or Fisher's exact test. $P$ - values $<0.05$ (two-tailed test) were considered indicative of statistically significant difference. Variables that showed a significant association on univariate analysis $(P<0.1)$ were included in the multivariable logistic regression model. Subsequently, the prediction model was constructed using backward stepwise selection algorithm with the Akaike information criterion. ${ }^{17-19}$

A nomogram was developed based on the model to predict the probability of LN-prRLN metastasis. The discriminative ability of the nomogram was measured using the concordance index (C-index). Unbiased $\mathrm{C}$-index was obtained through bootstrapping method (1000 resample) for internal validation to prevent overfitting. A larger $\mathrm{C}$-index implies greater accuracy in distinguishing the absence or presence of LN-prRLN metastasis. Calibration was evaluated by calibration plot and the Hosmer-Lemeshow test to interpret the proximity of the nomogram predicted risk with the actual risk. ${ }^{20,21}$ To evaluate the clinical usefulness of the nomogram, decision curve analysis (DCA) was conducted to calculate the net benefits at different threshold probabilities. $^{22}$ Statistical analysis was conducted using the R software version 3.5.1 (http://www.r-project.org).

\section{Results}

\section{Patient Characteristics}

A total of 592 patients were included in the analysis (Figure 1): 427 females and 165 males (female:male ratio, 2.58:1); mean age, $42.27 \pm 12.19$ years (range, 18-80). The characteristics of patients are summarized in Table 1 . The LN-prRLN metastatic rate was 19\% [95\% confidence interval (CI), 15.61-21.89\%]. In univariate analysis, no significant difference was observed between the LN-prRLN positive and LN-prRLN negative groups with respect to age, tumor location, or Hashimoto thyroiditis. The mean tumor size in LN-prRLN positive and LN-prRLN negative groups were $16.4 \mathrm{~mm}$ (SD 8.7) and $11.6 \mathrm{~mm}$ (SD 7.8), respectively $(P<0.001)$. The proportion of male patients in

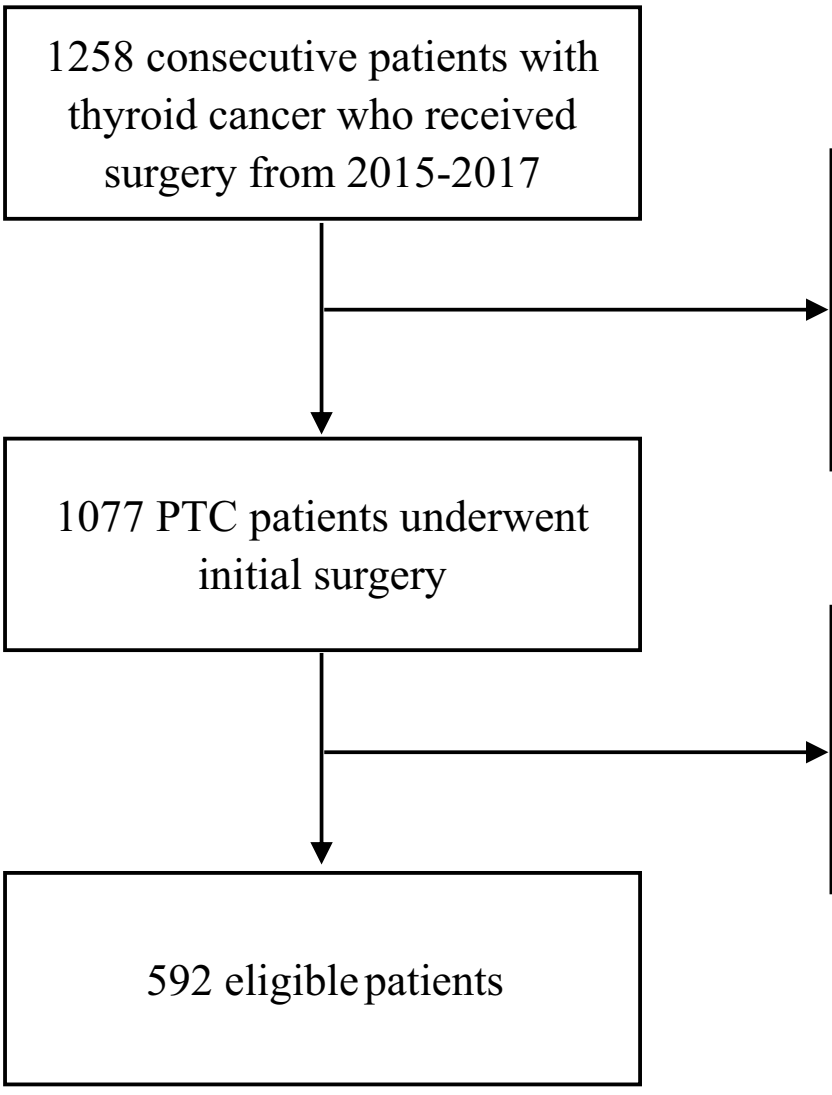

181 excluded

26 Histology not PTC

143 Re-operation

7 Distant metastasis

5 preoperative vocal cord paralysis

\section{5 excluded \\ 477 Did not meet inclusion criteria of right or bilateral lesions \\ 8 Missing clinical information}

Figure I Schematic illustration of the patient selection criteria. 
Table I Demographic And Clinicopathological Characteristics Of Patients With And Without LN-prRLN Metastasis

\begin{tabular}{|c|c|c|c|}
\hline \multirow[t]{2}{*}{ Characteristics } & \multicolumn{2}{|c|}{ LN-prRLN Metastasis, $n \%$} & \multirow[t]{2}{*}{$P$-value } \\
\hline & $\begin{array}{l}\text { Negative } \\
(n=48 I)\end{array}$ & $\begin{array}{l}\text { Positive } \\
(n=I I I)\end{array}$ & \\
\hline $\begin{array}{l}\text { Sex } \\
\qquad \text { Male } \\
\text { Female }\end{array}$ & $\begin{array}{l}122(25.4 \%) \\
359(74.6 \%)\end{array}$ & $\begin{array}{l}43(38.7 \%) \\
68(61.3 \%)\end{array}$ & 0.007 \\
\hline $\begin{array}{l}\text { Age (Mean } \pm S D \text {, years) } \\
\quad \leq 55 \\
>55\end{array}$ & $\begin{array}{l}42.6 \pm 12.2 \\
410(85.2 \%) \\
71(14.8 \%)\end{array}$ & $\begin{array}{l}41.0 \pm 12.0 \\
98(88.3 \%) \\
13(11.7 \%)\end{array}$ & $\begin{array}{l}0.215 \\
0.454\end{array}$ \\
\hline $\begin{array}{l}\text { Tumor size }(M \pm S D) \\
\quad \leq 10 \mathrm{~mm} \\
1 \mathrm{I}-20 \mathrm{~mm} \\
\geq 21 \mathrm{~mm}\end{array}$ & $\begin{array}{l}11.6 \pm 7.8 \\
293(60.9 \%) \\
149(31.0 \%) \\
39(8.1 \%)\end{array}$ & $\begin{array}{l}16.4 \pm 8.7 \\
37(33.3 \%) \\
55(49.6 \%) \\
19(17.1 \%)\end{array}$ & $\begin{array}{l}<0.001 \\
<0.001\end{array}$ \\
\hline $\begin{array}{l}\text { Location } \\
\text { Upper } \\
\text { Middle } \\
\text { Low }\end{array}$ & $\begin{array}{l}133(27.7 \%) \\
208(43.2 \%) \\
140(29.1 \%)\end{array}$ & $\begin{array}{l}34(30.6 \%) \\
46(4 I .4 \%) \\
31(28.0 \%)\end{array}$ & 0.821 \\
\hline $\begin{array}{l}\text { US-reported LN status } \\
\text { cNO } \\
\text { cNI }\end{array}$ & $\begin{array}{l}439 \text { (91.3\%) } \\
42(8.7 \%)\end{array}$ & $\begin{array}{l}71(64.0 \%) \\
40(36.0 \%)\end{array}$ & $<0.001$ \\
\hline $\begin{array}{l}\text { Multifocality } \\
\text { No } \\
\text { Yes }\end{array}$ & $\begin{array}{l}386(80.2 \%) \\
95 \text { (19.8\%) }\end{array}$ & $\begin{array}{l}71(64.0 \%) \\
40(36.0 \%)\end{array}$ & $<0.001$ \\
\hline $\begin{array}{l}\text { ETE } \\
\text { No } \\
\text { Yes }\end{array}$ & $\begin{array}{l}406 \text { (84.4\%) } \\
75 \text { (15.6\%) }\end{array}$ & $\begin{array}{l}75(67.6 \%) \\
36(32.4 \%)\end{array}$ & $<0.001$ \\
\hline $\begin{array}{l}\text { HT } \\
\text { No } \\
\text { Yes }\end{array}$ & $\begin{array}{l}385(80.0 \%) \\
96(20.0 \%)\end{array}$ & $\begin{array}{l}94 \text { (84.7\%) } \\
\text { I7 (I5.3\%) }\end{array}$ & 0.287 \\
\hline $\begin{array}{l}\text { Delphian LNM } \\
\text { No } \\
\text { Yes }\end{array}$ & $\begin{array}{l}42 \mid(87.5 \%) \\
60 \text { (12.5\%) }\end{array}$ & $\begin{array}{l}55(49.5 \%) \\
56(50.5 \%)\end{array}$ & $<0.001$ \\
\hline $\begin{array}{l}\text { Pretracheal LNM } \\
\text { No } \\
\text { Yes }\end{array}$ & $\begin{array}{l}317(65.9 \%) \\
164(34.1 \%)\end{array}$ & $\begin{array}{l}24(21.6 \%) \\
87(78.4 \%)\end{array}$ & $<0.001$ \\
\hline $\begin{array}{l}\text { Paratracheal LNM } \\
\text { No } \\
\text { Yes }\end{array}$ & $\begin{array}{l}303(63.0 \%) \\
178(37.0 \%)\end{array}$ & $\begin{array}{l}14(12.6 \%) \\
97(87.4 \%)\end{array}$ & $<0.001$ \\
\hline $\begin{array}{l}\mathrm{rCLNM}^{\mathrm{a}} \\
\text { No } \\
\text { Yes }\end{array}$ & $\begin{array}{l}232(48.2 \%) \\
249(51.8 \%)\end{array}$ & $\begin{array}{l}6(5.4 \%) \\
105(94.6 \%)\end{array}$ & $<0.001$ \\
\hline $\begin{array}{l}\text { LLNM } \\
\text { No } \\
\text { Yes }\end{array}$ & $\begin{array}{l}326(67.8 \%) \\
155(32.2 \%)\end{array}$ & $\begin{array}{l}23(20.7 \%) \\
88(79.3 \%)\end{array}$ & $<0.001$ \\
\hline
\end{tabular}

Note: ${ }^{a} \mathrm{LN}$-prRLN was excluded from rCLNM.

Abbreviations: LN-prRLN, lymph nodes posterior to the right recurrent laryngeal nerve; US, ultrasonography; ETE, extrathyroid extension; HT, Hashimoto thyroiditis; rCLNM, right central lymph node metastasis; LLNM, lateral lymph node metastasis. the LN-prRLN positive group was significantly higher than that in the LN-prRLN negative group $(38.7 \%$ vs $25.4 \%$, $P<0.001)$. The proportion of patients with multifocal lesions in the LN-prRLN positive group was significantly higher than that in the LN-prRLN negative group $(36.0 \%$ vs $19.8 \%, P<0.001)$. cN1 was more common in patients with positive LN-prRLN than in those with negative LN-prRLN $(36.0 \%$ vs $8.7 \%, P<0.001)$. LN-prRLN metastasis was associated with extrathyroid extension $(32.4 \%$ vs $15.6 \%$, $P<0.001$ ), Delphian lymph node metastasis (LNM; $50.5 \%$ vs $12.5 \%, P<0.001)$, pretracheal LNM ( $78.4 \%$ vs $34.1 \%$, $P<0.001)$, paratracheal LNM $(87.4 \%$ vs $37.0 \%, p<0.001)$, and lateral LNM ( $79.3 \%$ vs $32.2 \%, P<0.001)$. The negative rate of right central lymph node ( $\mathrm{rCLN})$ was $5.4 \%$ in the LN-prRLN positive group as against $48.2 \%$ in the LN-prRLN group $(P<0.001)$. The rCLN status showed $95 \%$ sensitivity, $48 \%$ specificity, $30 \%$ positive predictive value (PPV), and 97\% negative predictive value (NPV) for metastatic involvement of LN-prRLN.

On multivariate analyses, US-reported LN status ( $P=0.016)$, extrathyroid extension $(P=0.007)$ and Delphian LNM $(P=0.001)$, number of metastatic pretracheal lymph nodes $(P=0.005)$, and paratracheal lymph nodes $(P<0.001)$ were independent predictors of LN-prRLN metastasis (Table 2). The variable "rCLN metastasis" was excluded from multivariate analysis because of collinearity.

\section{Nomogram Development}

A nomogram incorporating the above 5 predictors is presented in Figure 2. The C-index for the nomogram was 0.87 (95\% CI, $0.84-0.91)$ and 0.86 by bootstrapping validation, which demonstrated good discriminative ability. The calibration plot revealed excellent agreement between the prediction and the actual observation (Figure 3). Hosmer-Lemeshow test yielded a nonsignificant $P=0.55$, indicating no departure from a good fit. DCA suggested that if the threshold probability is higher than $10 \%$, use of this nomogram to predict LN-prRLN metastasis would yield greater benefits than that accruing from a "treat-all" or "treat-none" strategy (Figure 4).

\section{Discussion}

Accurate evaluation of the nodal status is an essential prerequisite for personalized therapy of patients with PTC. Current methods for the evaluation of the LN-prRLN status have several limitations. In the present study, US-reported LN status, extrathyroid extension, US-reported LN status, Delphian LNM, and the number of metastatic pretracheal 
Table 2 Results Of Multivariate Logistic Regression Analysis Showing Predictors Of LN-prRLN Metastasis

\begin{tabular}{|l|l|l|}
\hline Variables & OR (95\% Cl) & P-value \\
\hline US-reported LN status (cNI vs. cN0) & $2.179(1.156-4.107)$ & 0.016 \\
Extrathyroid extension (yes vs. no) & $2.232(1.240-4.017)$ & 0.007 \\
Delphian LNM (yes vs. no) & $2.580(1.466-4.54 I)$ & 0.001 \\
\hline No. of pretracheal LNM & & \\
0 & I (reference) & \\
I-3 & $2.499(1.376-4.54 I)$ & 0.003 \\
$>3$ & $3.558(1.467-8.626)$ & 0.005 \\
\hline No. of paratracheal LNM & & \\
0 & $I$ (reference) & \\
I-3 & $4.866(2.789-8.490)$ & $<0.00 \mathrm{I}$ \\
$>3$ & $2 I .078(7.305-60.8 I 7)$ & $<0.00 \mathrm{I}$ \\
\hline
\end{tabular}

Abbreviations: US, ultrasonography; LNM, lymph node metastasis; OR, odds ratio; $\mathrm{Cl}$, confidence interval.

and paratracheal lymph nodes were independent predictors of LN-prRLN metastasis. In previous studies, these 5 factors were associated with poor prognosis of patients with PTC. ${ }^{23,24}$ Using these predictive factors, we developed and internally validated a nomogram to predict the likelihood of LN-prRLN metastasis. The model showed good discriminative ability and calibration. Our findings signify that readily available preoperative and intraoperative clinicopathological

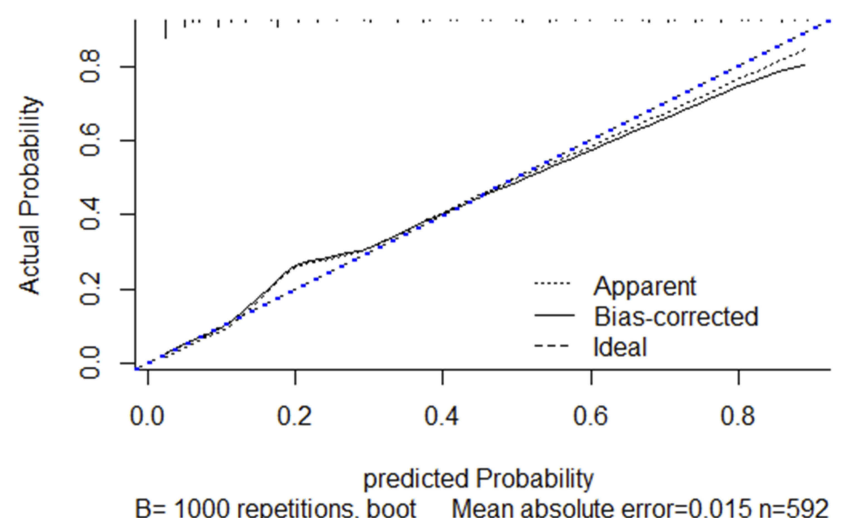

Figure 3 Calibration plot of the nomogram. The calibration curve depicts the calibration of model in terms of the agreement between the predicted risks of LNprRLN metastasis and observed outcomes of LN-prRLN metastasis. The $x$-axis represents the predicted LN-prRLN metastasis risk. The $y$-axis represents the actual LN-prRLN metastasis rate. The diagonal dotted blue line represents an ideal calibration model in which the predicted probabilities are identical to the actual outcomes. The dotted line represents the predictive performance of the nomogram; the closer the fit of the dotted line to the ideal line, the better the prediction. The black line represents the bias corrected.

features can be used to estimate the risk of LN-prRLN metastasis and facilitate surgical decision-making with respect to selective lymphadenectomy.

Consistent with the previous study, ${ }^{13}$ extrathyroid extension was an independent risk factor for LN-prRLN metastasis in this study; this reflects the perspective that
Points

US_reported_LN_status

ETE

Delphian_LNM

No.of_pretracheal_LNM

No.of_paratracheal_LNM

\section{Total Points}

\section{Risk of LN-prRLN metastasis}
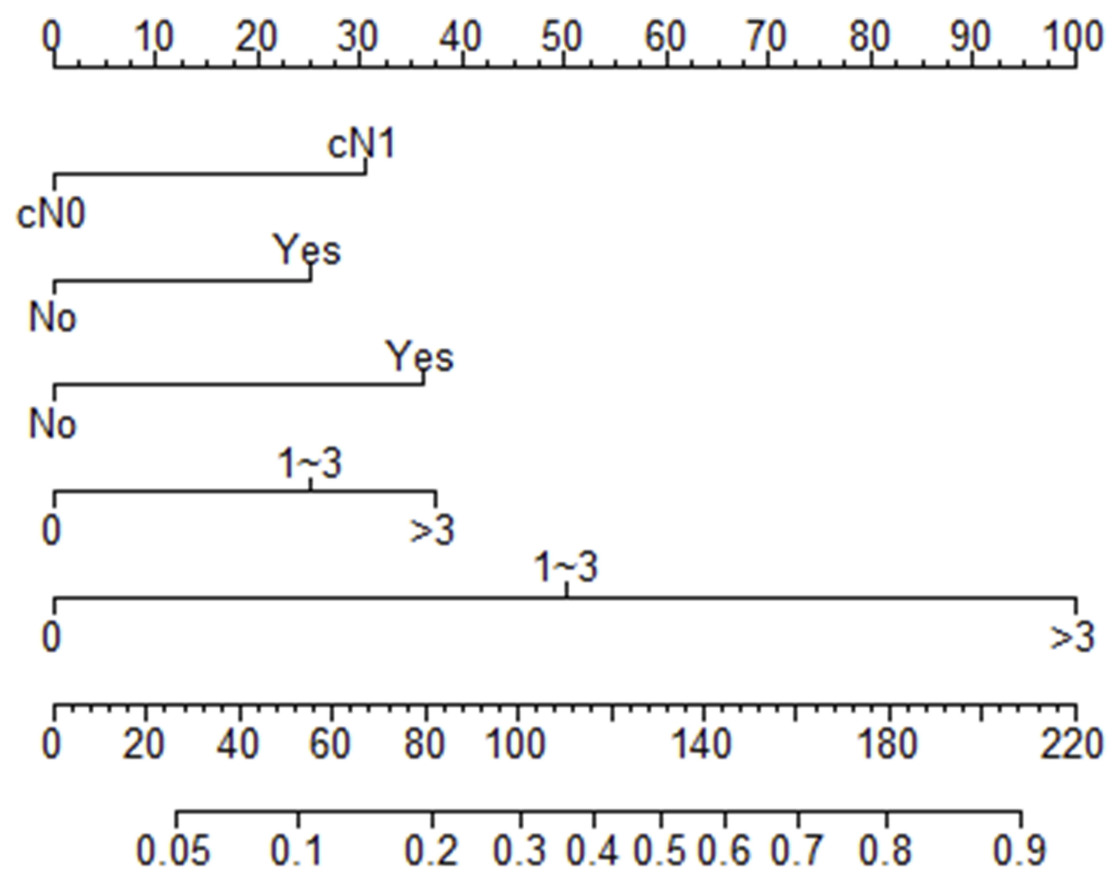

Figure 2 Nomogram for prediction of LN-prRLN metastases in patients with PTC. A line is drawn straight up to the point axis that corresponds with each patient variable to obtain the points. The sum of these points is located on the total score points axis. Lastly, a line is drawn downwards to the risk axis to determine the likelihood of $L N-$ prRLN metastases.

Abbreviations: US-reported LN status, ultrasound-reported lymph node status; ETE, extrathyroidal extension; Delphian LNM, Delphian lymph node metastasis; No. of pretracheal LNM, number of pretracheal lymph node metastasis; No. of paratracheal LNM, number of paratracheal lymph node metastasis. 


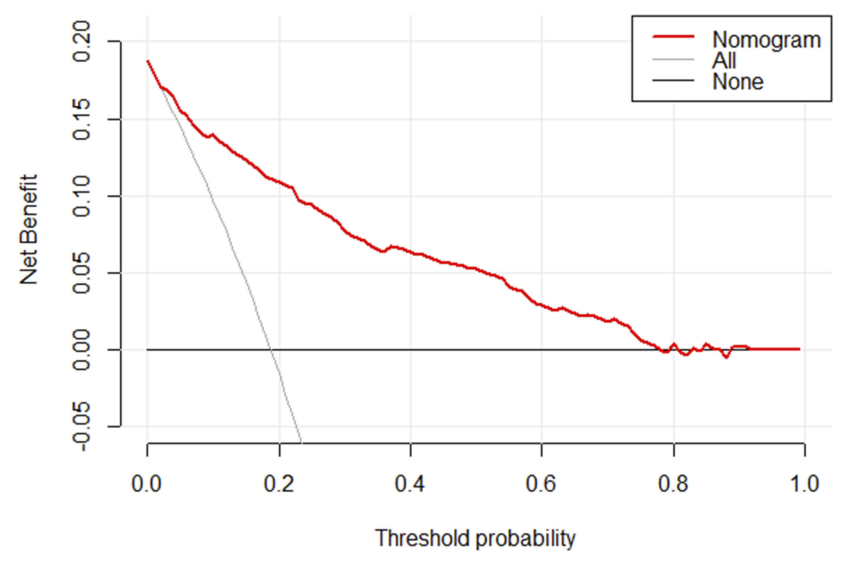

Figure 4 Decision curve analysis for the nomogram. The $y$-axis measures the net benefit. The $x$-axis represents the threshold probability. The red solid line represents the nomogram. The gray line represents the hypothesis that all patients had LN-prRLN metastasis. The black solid line represents the hypothesis that none of the patients had LN-prRLN metastasis. The decision curve indicates that when the threshold probability is $>10.0 \%$, use of this predictive model would accrue greater benefit than that accruing from a treat-all or treat-none strategy.

the presence of extrathyroid extension is associated with more aggressive disease and poorer outcomes. ${ }^{25}$ USreported LN status was chosen as a candidate variable in the multivariate analysis in light of its association with the actual lymph node status. As shown in the nomogram, cN1 was more likely to be associated with LN-prRLN metastasis than cN0.

Luo et al recently proposed a prediction model ${ }^{26}$ However, their model was based on preoperative clinicopathologic risk factors. It is unclear whether incorporation of intraoperative clinicopathologic characteristics can improve the accuracy of prediction. The right central compartment comprised of Delphian, pretracheal, LN-prRLN, and paratracheal lymph node groups. The Delphian lymph node (prelaryngeal) is located above the isthmus between the thyroid cartilage and cricoid. Studies have also shown that positive Delphian lymph node in PTC implies heavier cancer burden and greater nodal involvement. ${ }^{27}$ In our study, Delphian LNM was a predictor of LN-prRLN metastasis on univariate and multivariate analysis. We categorized the number of metastatic pretracheal lymph nodes and paratracheal lymph nodes as $0,1-3$, and $>3$. The number of metastatic pretracheal lymph nodes and paratracheal lymph nodes both showed a significant association with LN-prRLN metastasis. It is noteworthy that paratracheal LNM was found to be an extremely strong predictor of LN-prRLN metastasis, which suggests that involvement of paratracheal LNM signifies a greater risk of LN-prRLN metastasis because of the anatomical proximity. In addition, according to Zhang et al, the risk of LN-prRLN metastasis with $\geq 3$ lateral metastatic nodes was three times higher than that with $<3$ metastatic nodes. ${ }^{28}$ Our data showed that lateral LNM is related to LNprRLN metastasis. We still excluded lateral lymph node metastasis from the prediction model because the status is unknown prior to central neck lymph node dissection. However, it is important to perform rigorous screening of lateral cervical lymph nodes with preoperative ultrasonography.

The 2015 American Thyroid Association (ATA) guidelines suggested a selective approach to central compartment neck dissection. ${ }^{8}$ In our series, only $2.5 \%$ (6/238) patients showed LN-prRLN metastasis in the absence of rCLN metastasis (NPVs: 97\%); based on this finding, we favor conservative neck dissection in the absence of suspicious metastatic lymph nodes. Indeed, it is relatively safe to excise the Delphian, pretracheal, paratracheal lymph nodes compared with the LN-prRLN dissection. Intraoperative frozen section examination is a reliable technique that has been widely used for patients with PTC; it allows for accurate intraoperative diagnosis of nodal status of each subgroup in real-time. ${ }^{29,30}$ Thus, as the preoperative US-reported LN status is routinely reported before operation in clinical practice, we recommend reasonable surgical exploration to obtain intraoperative information through precision zoning and rapid intraoperative frozen section examination; all of these readily available clinicopathologic features can be used to build a nomogram for individual prediction of LN-prRLN metastasis. The nomogram showed favorable discriminative ability and good calibration curve. Moreover, decision curves showed that if the threshold probability is higher than $10 \%$, use of the nomogram to predict lymph node status provides greater net benefit than that accruing from a "treat-all" or "treat-none" strategy. ${ }^{22}$ Therefore, the prediction model can assist surgeons in making a precise prediction of the LN-prRLN metastasis and to decide to continue or stop the dissection during dissection of the central compartment of the neck.

Several studies have reported independent risk factors for LN-prRLN metastasis; ${ }^{12,28}$ however, there was much variability with respect to the inclusion and exclusion criteria used in the individual studies. To the best of our knowledge, few studies have reported about a convenient and accurate tool for the prediction of LN-prRLN metastasis. In the present study, we developed a nomogram by incorporating both preoperative clinicopathologic variables and intraoperative frozen section examination and validated its accuracy to predict the risk of LN-prRLN metastasis in patients with PTC.

There are limitations of this study. In this study, we included all patients from July 2015 to December 2017, 
which represents the real-world experience of our hospital. Unfortunately, it was a single-center retrospective study with a relatively small sample size, although rigorous validation with bootstrapped calibration (1000 resample) and optimism-corrected estimates, the nomogram should be externally validated in large multi-institutional databases to test its applicability to different populations. ${ }^{17}$ It is hoped that a future randomized controlled trial will determine the application value of the nomogram.

\section{Conclusion}

In summary, intraoperative clinicopathologic variables were independent predictors of LN-prRLN metastasis in patients with PTC. By incorporating readily available preoperative information and intraoperative frozen section examination, we developed a nomogram for individualized risk prediction of LN-prRLN metastasis. The prediction model may be used to guide intraoperative decision making for tailored surgical intervention.

\section{Ethical Approval And Informed Consent}

This study was in accordance with the Declaration of Helsinki and approved by the Institutional Review Board of the First Affiliated Hospital of Chongqing Medical University. Written informed consent was obtained from all individual participants included in the study.

\section{Disclosure}

The authors report no conflicts of interest in this work.

\section{References}

1. Bray F, Ferlay J, Soerjomataram I, Siegel RL, Torre LA, Jemal A. Global cancer statistics 2018: GLOBOCAN estimates of incidence and mortality worldwide for 36 cancers in 185 countries. CA Cancer $J$ Clin. 2018;68(6):394-424. doi:10.3322/caac.v68.6

2. Global Burden of Disease Cancer C, Fitzmaurice C, Akinyemiju TF, Al Lami FH, et al. Global, regional, and National Cancer Incidence, mortality, years of life lost, years lived with disability, and disabilityadjusted life-years for 29 cancer groups, 1990 to 2016: a systematic analysis for the global burden of disease study. JAMA Oncol. 2018;4 (11):1553-1568. doi:10.1001/jamaoncol.2018.2706

3. Mulla M, Schulte KM. Central cervical lymph node metastases in papillary thyroid cancer: a systematic review of imaging-guided and prophylactic removal of the central compartment. Clin Endocrinol (Oxf). 2012;76(1):131-136. doi:10.1111/j.1365-2265. 2011.04162.x

4. Leboulleux S, Rubino C, Baudin E, et al. Prognostic factors for persistent or recurrent disease of papillary thyroid carcinoma with neck lymph node metastases and/or tumor extension beyond the thyroid capsule at initial diagnosis. J Clin Endocrinol Metab. 2005;90 (10):5723-5729. doi:10.1210/jc.2005-0285
5. Hartl DM, Leboulleux S, Al Ghuzlan A, et al. Optimization of staging of the neck with prophylactic central and lateral neck dissection for papillary thyroid carcinoma. Ann Surg. 2012;255(4):777783. doi:10.1097/SLA.0b013e31824b7b68

6. Suh YJ, Kwon H, Kim SJ, et al. Factors affecting the locoregional recurrence of conventional papillary thyroid carcinoma after surgery: a retrospective analysis of 3381 patients. Ann Surg Oncol. 2015;22 (11):3543-3549. doi:10.1245/s10434-015-4448-9

7. Young S, Harari A, Smooke-Praw S, Ituarte PH, Yeh MW. Effect of reoperation on outcomes in papillary thyroid cancer. Surgery. 2013;154 (6):1354-1361. (discussion 1361-1352). doi:10.1016/j.surg.2013.06.043

8. Haugen BR, Alexander EK, Bible KC, et al. 2015 American Thyroid Association management guidelines for adult patients with thyroid nodules and differentiated thyroid cancer: the American Thyroid Association Guidelines task force on thyroid nodules and differentiated thyroid cancer. Thyroid. 2016;26(1):1-133. doi:10.1089/thy.2015.0020

9. Wang TS, Sosa JA. Thyroid surgery for differentiated thyroid cancer - recent advances and future directions. Nat Rev Endocrinol. 2018;14 (11):670-683. doi:10.1038/s41574-018-0080-7

10. Miller JE, Al-Attar NC, Brown OH, et al. Location and causation of residual lymph node metastasis after surgical treatment of regionally advanced differentiated thyroid cancer. Thyroid. 2018;28(5):593-600. doi:10.1089/thy.2017.0434

11. Kouvaraki MA, Shapiro SE, Fornage BD, et al. Role of preoperative ultrasonography in the surgical management of patients with thyroid cancer. Surgery. 2003;134(6):946-954. doi:10.1016/S0039-6060(03) 00424-0

12. Bae SY, Yang JH, Choi MY, Choe JH, Kim JH, Kim JS. Right paraesophageal lymph node dissection in papillary thyroid carcinoma. Ann Surg Oncol. 2012;19(3):996-1000. doi:10.1245/s10434-011-2144-y

13. Ito Y, Fukushima M, Higashiyama T, et al. Incidence and predictors of right paraesophageal lymph node metastasis of N0 papillary thyroid carcinoma located in the right lobe. Endocr J. 2013;60(3):389392. doi:10.1507/endocrj.EJ12-0362

14. Lee BJ, Lee JC, Wang SG, Kim YK, Kim IJ, Son SM. Metastasis of right upper para-esophageal lymph nodes in central compartment lymph node dissection of papillary thyroid cancer. World J Surg. 2009;33(10):2094-2098. doi:10.1007/s00268-009-0149-5

15. Pinyi Z, Bin Z, Jianlong B, Yao L, Weifeng Z. Risk factors and clinical indication of metastasis to lymph nodes posterior to right recurrent laryngeal nerve in papillary thyroid carcinoma: a single-center study in China. Head Neck. 2014;36(9):1335-1342. doi:10.1002/hed.23451

16. $\mathrm{Hu} \mathrm{D,} \mathrm{Zhou} \mathrm{J,} \mathrm{He} \mathrm{W,} \mathrm{et} \mathrm{al.} \mathrm{Risk} \mathrm{factors} \mathrm{of} \mathrm{lateral} \mathrm{lymph} \mathrm{node}$ metastasis in cN0 papillary thyroid carcinoma. World J Surg Oncol. 2018;16(1):30. doi:10.1186/s12957-018-1336-3

17. Collins GS, Reitsma JB, Altman DG, Moons KG. Transparent reporting of a multivariable prediction model for individual prognosis or diagnosis (TRIPOD): the TRIPOD statement. Br J Surg. 2015;102 (3):148-158. doi:10.1002/bjs.2015.102.issue-3

18. Lin DZ, Qu N, Shi RL, Lu ZW, Ji QH, Wu WL. Risk prediction and clinical model building for lymph node metastasis in papillary thyroid microcarcinoma. Onco Targets Ther. 2016;9:5307-5316. doi:10.2147/OTT.S107913

19. Wang Y, Li J, Xia Y, et al. Prognostic nomogram for intrahepatic cholangiocarcinoma after partial hepatectomy. J Clin Oncol. 2013;31 (9):1188-1195. doi:10.1200/JCO.2012.41.5984

20. Iasonos A, Schrag D, Raj GV, Panageas KS. How to build and interpret a nomogram for cancer prognosis. J Clin Oncol. 2008;26 (8):1364-1370. doi:10.1200/JCO.2007.12.9791

21. Balachandran VP, Gonen M, Smith JJ, DeMatteo RP. Nomograms in oncology: more than meets the eye. Lancet Oncol. 2015;16(4):e173e180. doi:10.1016/S1470-2045(14)71116-7

22. Vickers AJ, Cronin AM, Elkin EB, Gonen M. Extensions to decision curve analysis, a novel method for evaluating diagnostic tests, prediction models and molecular markers. BMC Med Inform Decis Mak. 2008;8:53. doi:10.1186/1472-6947-8-53 
23. Nixon IJ, Wang LY, Palmer FL, et al. The impact of nodal status on outcome in older patients with papillary thyroid cancer. Surgery. 2014;156(1):137-146. doi:10.1016/j.surg.2014.03.027

24. Chen W, Lei J, You J, et al. Predictive factors and prognosis for recurrent laryngeal nerve invasion in papillary thyroid carcinoma. Onco Targets Ther. 2017;10:4485-4491. doi:10.2147/OTT

25. Londero SC, Krogdahl A, Bastholt L, et al. Papillary thyroid carcinoma in Denmark, 1996-2008: outcome and evaluation of established prognostic scoring systems in a prospective national cohort. Thyroid. 2015;25(1):78-84. doi:10.1089/thy.2014.0294

26. Luo Y, Xu XC, Shen J, et al. Model of lymph node metastasis posterior to the right recurrent laryngeal nerve in papillary thyroid carcinoma. Cancer Manag Res. 2018;10:2449-2455. doi:10.2147/CMAR.S167997

27. Iyer NG, Kumar A, Nixon IJ, et al. Incidence and significance of delphian node metastasis in papillary thyroid cancer. Ann Surg. 2011;253(5):988-991. doi:10.1097/SLA.0b013e31821219ca
28. Zhang L, Liu H, Xie Y, et al. Risk factors and indication for dissection of right paraesophageal lymph node metastasis in papillary thyroid carcinoma. Eur J Surg Oncol. 2016;42(1):81-86. doi:10.1016/j. ejso.2015.10.011

29. Raffaelli M, De Crea C, Sessa L, Fadda G, Bellantone C, Lombardi CP. Ipsilateral central neck dissection plus frozen section examination versus prophylactic bilateral central neck dissection in $\mathrm{cN} 0$ papillary thyroid carcinoma. Ann Surg Oncol. 2015;22(7):2302-2308. doi:10.1245/ s10434-015-4383-9

30. Giordano D, Valcavi R, Thompson GB, et al. Complications of central neck dissection in patients with papillary thyroid carcinoma: results of a study on 1087 patients and review of the literature. Thyroid. 2012;22(9):911-917. doi:10.1089/thy.2012.0011

\section{Publish your work in this journal}

OncoTargets and Therapy is an international, peer-reviewed, open access journal focusing on the pathological basis of all cancers, potential targets for therapy and treatment protocols employed to improve the management of cancer patients. The journal also focuses on the impact of management programs and new therapeutic agents and protocols on patient perspectives such as quality of life, adherence and satisfaction. The manuscript management system is completely online and includes a very quick and fair peer-review system, which is all easy to use. Visit http://www.dovepress.com/ testimonials.php to read real quotes from published authors. 